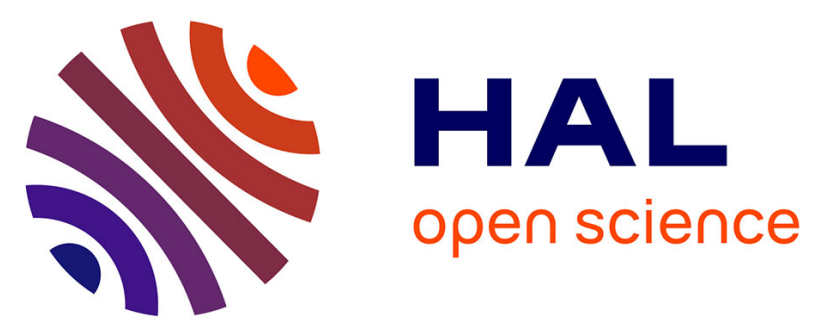

\title{
Oedometric-like setup for the study of water transport in porous media by quasi-elastic neutron scattering
}

Julie Wolanin, Jérôme Giraud, Claude Payre, Marianne Benoit, Claire

Antonelli, Damien Quemener, Iliass Tahiri, Matthieu Vandamme, Jean-Marc Zanotti, Marie Plazanet

\section{To cite this version:}

Julie Wolanin, Jérôme Giraud, Claude Payre, Marianne Benoit, Claire Antonelli, et al.. Oedometriclike setup for the study of water transport in porous media by quasi-elastic neutron scattering. Review of Scientific Instruments, 2021, 92 (2), pp.024106. 10.1063/5.0030297 . hal-03162209

\section{HAL Id: hal-03162209 \\ https://hal.umontpellier.fr/hal-03162209}

Submitted on 23 Nov 2021

HAL is a multi-disciplinary open access archive for the deposit and dissemination of scientific research documents, whether they are published or not. The documents may come from teaching and research institutions in France or abroad, or from public or private research centers.
L'archive ouverte pluridisciplinaire HAL, est destinée au dépôt et à la diffusion de documents scientifiques de niveau recherche, publiés ou non, émanant des établissements d'enseignement et de recherche français ou étrangers, des laboratoires publics ou privés. 
Oedometric-like setup for the study of water transport in porous media by quasi-elastic neutron scattering

Julie Wolanin ${ }^{1, a)}$, Jérôme Giraud ${ }^{1}$, Claude Payre ${ }^{2}$, Marianne Benoit ${ }^{3}$, Claire Antonelli ${ }^{3}$, Damien Quemener ${ }^{3}$, Iliass Tahiri ${ }^{4}$, Matthieu Vandamme ${ }^{4}$, Jean-Marc Zanotti ${ }^{5}$ and Marie Plazanet ${ }^{1, a)}$

${ }^{1}$ Univ. Grenoble Alpes, CNRS, LIPhy, 38000 Grenoble, France

${ }^{2}$ Institut Laue Langevin, 38042 Grenoble, France

${ }^{3}$ Institut Européen des Membranes, IEM-UMR 5635, Univ Montpellier, ENSCM, CNRS,34090

Montpellier, France

${ }^{4}$ Laboratoire Navier, Ecole des Ponts ParisTech, Univ Gustave Eiffel, CNRS, 77420 Marne-laVallée, France

${ }^{5}$ Laboratoire Léon Brillouin, CEA, CNRS, Université Paris-Saclay, CEA Saclay, 91191 Gif-surYvette Cedex, France

a) Authors to whom correspondence should be addressed: julie.wolanin@univ-grenoble-alpes.fr and marie.plazanet@univ-grenoble-alpes.fr

\begin{abstract}
In comparison to condensed matter, soft matter is subject to several interplaying effects (surface heterogeneities, swelling effect...) that influence transport at the nanoscale. In consequence, transport in soft and compliant materials is coupled to adsorption and deformation phenomena. The permeance of the material, i.e. the response of the material to a pressure gradient, is dependent on the temperature, the chemical potential and the external constraint. Therefore, the characterization of water dynamics in soft porous materials, that we address here, becomes much more complex. In this paper, the development of an original setup for scattering measurements of a radiation in the transmitted geometry in oedometric conditions is described. A specially designed cell enables a uniaxial compression of the investigated material, PIM-1 (Polymers of Intrinsic Microporosity), in the direction perpendicular to the applied hydraulic pressure gradient (up to 120 bars). High pressure boosting of the circulating water is performed with a commercially available high-pressure pump Karcher. This particular setup is adapted to Quasi-elastic neutron scattering technique, which enables to probe diffusion and relaxation phenomena with characteristic times of $10^{-9}-10^{-12} \mathrm{~s}$. Moreover, it can easily be modified for other scattering techniques.
\end{abstract}

Text

\title{
1. Introduction
}

Transport of water in soft porous materials is relevant to a broad range of applications such as ultrafiltration, reverse osmosis, swelling of wood materials or energy related processes. In the past few years, the dynamics of water confined in nanospaces has been particularly investigated in hard materials (such as porous silica ${ }^{1-4}$, silica gel ${ }^{5}$, vycor glasses ${ }^{6}$, alumina gel ${ }^{7}, \ldots$ ). However, the water transport in soft matter is still not clear as additional non-negligible effects have to be considered (surface heterogeneities, host deformation...). Although they cannot be ignored in the case of soft environment, such effects are usually not considered when describing water transport in porous materials. Indeed, in the literature, studies only reported microscopic diffusion of water in soft matter 
${ }^{8}$ but not under transport conditions, and rarely considering (exclusively with computational tools ${ }^{9}$ ) the local or global deformations of the host.

The proposed work describes a suitable setup specially designed to investigate the coupling between water transport and host deformation in soft porous materials, under relevant conditions of uniaxial compression (linear deformation of the material, $<10 \%$ ) and hydraulic pressure gradient (up to 120 bars for technical reasons). For this purpose, quasi-elastic neutron scattering (QENS) experiments are the most appropriate to be performed. Indeed, QENS is the only tool giving information on single-particle diffusive motions under nanometric confinement at the molecular scale. Several information such as self and collective diffusion coefficients, diffusion mechanisms (long-range or jump translational diffusions, molecular reorientations...) as well as the number of water molecules trapped into the walls and immobile over the investigated time scale can be obtained $7,10,11$

In order to perform QENS experiments in relevant conditions for transport in soft materials, we propose here an innovative set up using the inexpensive available Karcher K7 to control the hydraulic pressure gradient, connected to a newly designed aluminum pressure cell (clamp-type cell). To the best of our knowledge, only few experimental setups have used a Karcher to pressurize water ${ }^{12-14}$, most of the time for high pressure rinsing systems studies. After presenting the details of the setup and characteristics of the materials used, we also prove the feasibility of the proposed experiment by performing QENS measurements on the hydrophobic porous material PIM-1 (Polymers of Intrinsic Microporosity) which presents an interconnected porosity at the nanometre scale $(<2 \mathrm{~nm})^{15}$. We emphasize that the application of the presented setup can be easily extended to other experimental techniques. For example, synchrotron experiments could be performed to characterize changes in the film structure in such conditions, by using an equivalent cell to the one designed for QENS but adapted for X-rays measurements with beryllium windows.

\section{High-pressure set-up}

\subsection{Homemade high-pressure cell for QENS experiments}

Our high-pressure cell (Figure 1) made of 7075 aluminum alloy has been designed to simultaneously investigate the effect of a hydraulic pressure gradient and a mechanical stress applied on a thin $(\sim 300 \mu \mathrm{m})$ porous film. 7075 aluminum alloy (composed of $\sim 90.0 \mathrm{wt} \% \mathrm{Al}$ and small percentages of $\mathrm{Zn}, \mathrm{Mg}, \mathrm{Cr}$, and $\mathrm{Cu}$ ) is one of the strongest aluminum alloys and is adapted for QENS experiments as aluminum has low neutron absorption cross section.

The cell is made of two assembled parts. The top (Figure 2a) of the cell is $10 \mathrm{~mm}$ thick and the bottom (Figure 2b) $14 \mathrm{~mm}$ thick. The thicknesses have been chosen as a compromise between high transmission for neutrons and low mechanical deformation of the cell under hydraulic pressure. For this reason, the bottom plate is excavated in the center on its external side. 


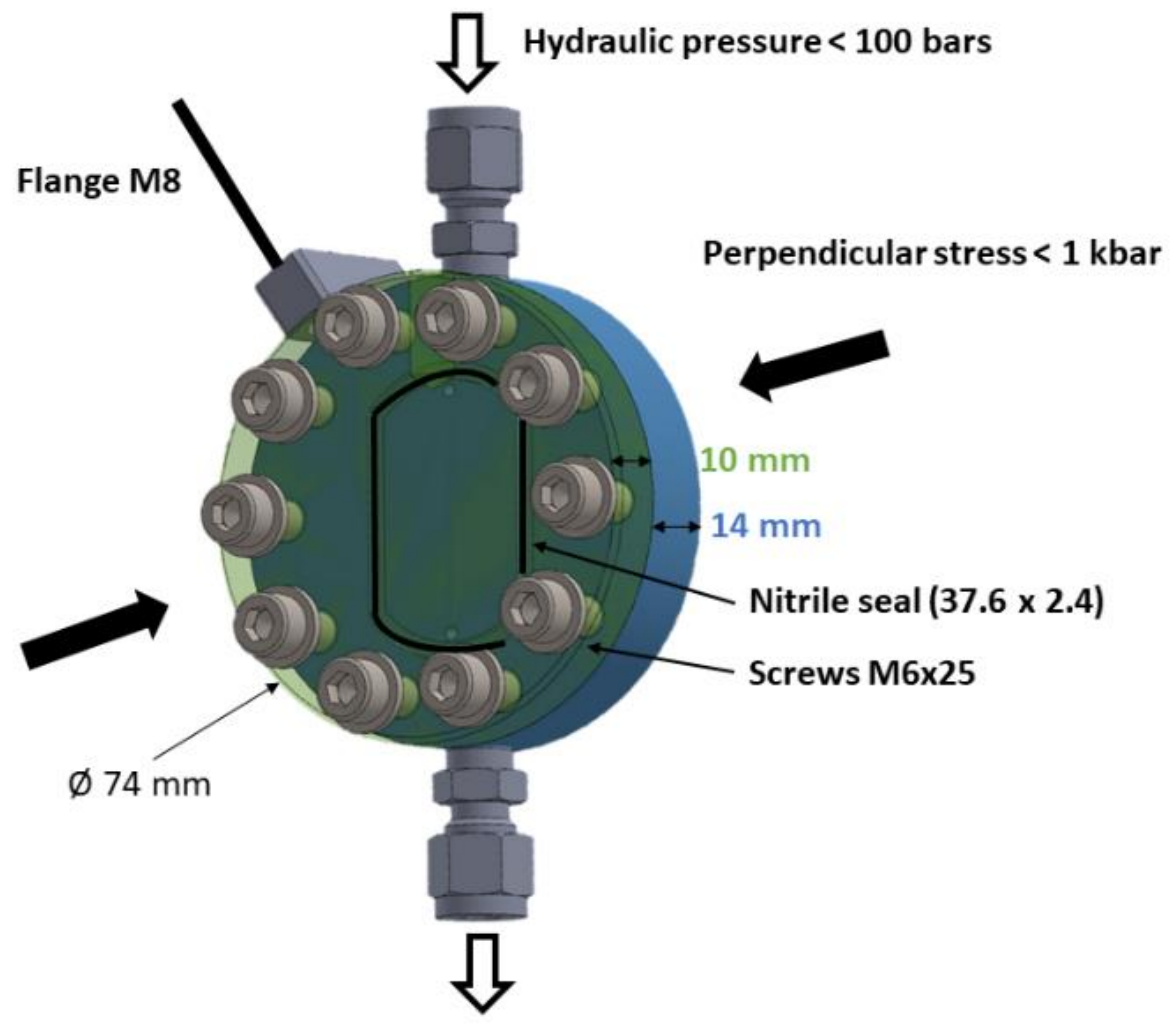

Figure 1: High-pressure cell sketch

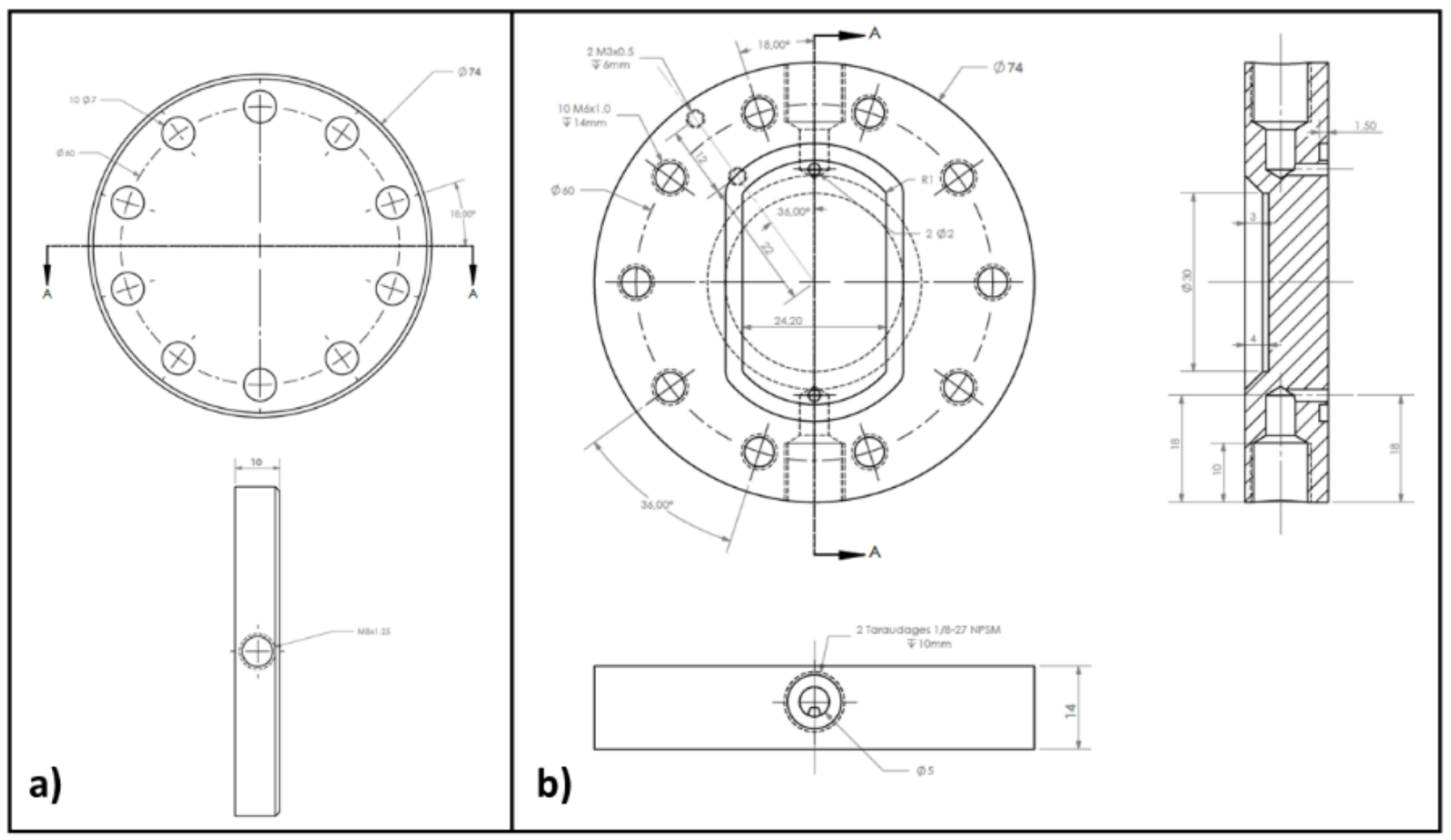

Figure 2: Drawings of the top (a) and bottom (b) parts of the cell

The mechanical stress is provided by a clamp-like system thanks to 10 screws. The uniaxial compression of the film is applied ex-situ with a torque wrench ensuring a proper tightening level (to a fixed torque value) of the screws in the direction perpendicular to the applied hydraulic pressure gradient. The Karcher (model K7 full control) is used to control the water flow. 
The investigated soft porous film, PIM-1 ( $300 \mu \mathrm{m}$ thick), is sandwiched between the two flat plates (no excavation is made for the sample) and surrounded by a nitrile seal. The O-ring enables water flow by achieving a watertight joint between the two parts of the cell, and could hold up to 500 bars hydraulic pressure. Moreover, the nitrile seal will also compensate for the sample thickness and adjust the mechanical constraint, being more or less compressed according to the initial uniaxial compression applied.

\subsection{Cell deformation during operations}

The cell deformation upon water flow is important to be characterized since it can change the final compression of the sample. We initially measured the external deformation of the centre of the cell. The measurements were performed with two dial Mitutoyo test indicators (lever type) at three different compression levels (tightening of the screws) and at 100 bars hydrostatic pressure. Both, top (flat side) and bottom (excavated side) part were considered. The results are presented in Table I.

The highest deformation measured at $1 \mathrm{Nm}$ is due to the weak sealing of the two plates with the screws which can loosen under water pressure. As the investigated sample is $\sim 300 \mu \mathrm{m}$ thick, we have to consider the $10 \mu \mathrm{m}$ cell deformation while quantifying the film deformation. We note that at 100 bars hydrostatic pressure, the deformation is reversible.

Table I: Measurements of the deformation of the center of the cell (no sample inside) using fluorinert as solvent at 100 bars of hydrostatic pressure

\begin{tabular}{|c|c|c|c|}
\hline $\begin{array}{c}\text { Torque } \\
{[\mathbf{N m}]}\end{array}$ & $\mathbf{1}$ & $\mathbf{5}$ & $\mathbf{1 0}$ \\
\hline Top $( \pm 0.01 \mathrm{~mm})$ & $0.04 \mathrm{~mm}$ & $0.01 \mathrm{~mm}$ & $0.01 \mathrm{~mm}$ \\
\hline Bottom $( \pm 0.01 \mathrm{~mm})$ & $0.02 \mathrm{~mm}$ & $0.00 \mathrm{~mm}$ & $0.00 \mathrm{~mm}$ \\
\hline
\end{tabular}

\subsection{Measurement of the soft film compression}

Since the uniaxial compression is applied simultaneously on the sample and on the nitrile seal, the exact load applied on the sample needs to be independently characterized. This was performed with two different subminiature industrial load sensors (from Omega Engineering Limited) inserted in a compression load cell specially designed for the measurement. The load range accessible depends on the sensor capacity; the range $1 \mathrm{~N}$ to $5 \mathrm{kN}$ was investigated with the LCMKD-5KN sensor while the $5 \mathrm{kN}$ to $50 \mathrm{kN}$ range with the LCM307-50KN one. The sketch of the cell including the load sensor LCMKD-5KN placement is shown in Figure 3a. The top of the cell remains unchanged (see Figure $2 \mathrm{a}$ ), only the bottom part includes some modifications according to the sizes of the sensors used.

We note that an aluminium flat plate was placed on the load sensor during measurements, located in the hole (Figure 3c). Indeed, this flat plate which has the same dimensions as the investigated sample $(20 \times 30 \mathrm{~mm})$ but $10 \mathrm{~mm}$ thick, avoids the soft film to be damaged and deformed by the sensible part of the load sensor $(0.76 \mathrm{~mm}$ height and $6.1 \mathrm{~mm}$ diameter for example for the LCMKD-5KN), without introducing additional friction. The strain indicator DP400S (from Omega Engineering 
Limited) displays the compression load values. The measurements were performed under dry conditions, without water flow nor hydrostatic pressure with the PIM-1 sample.
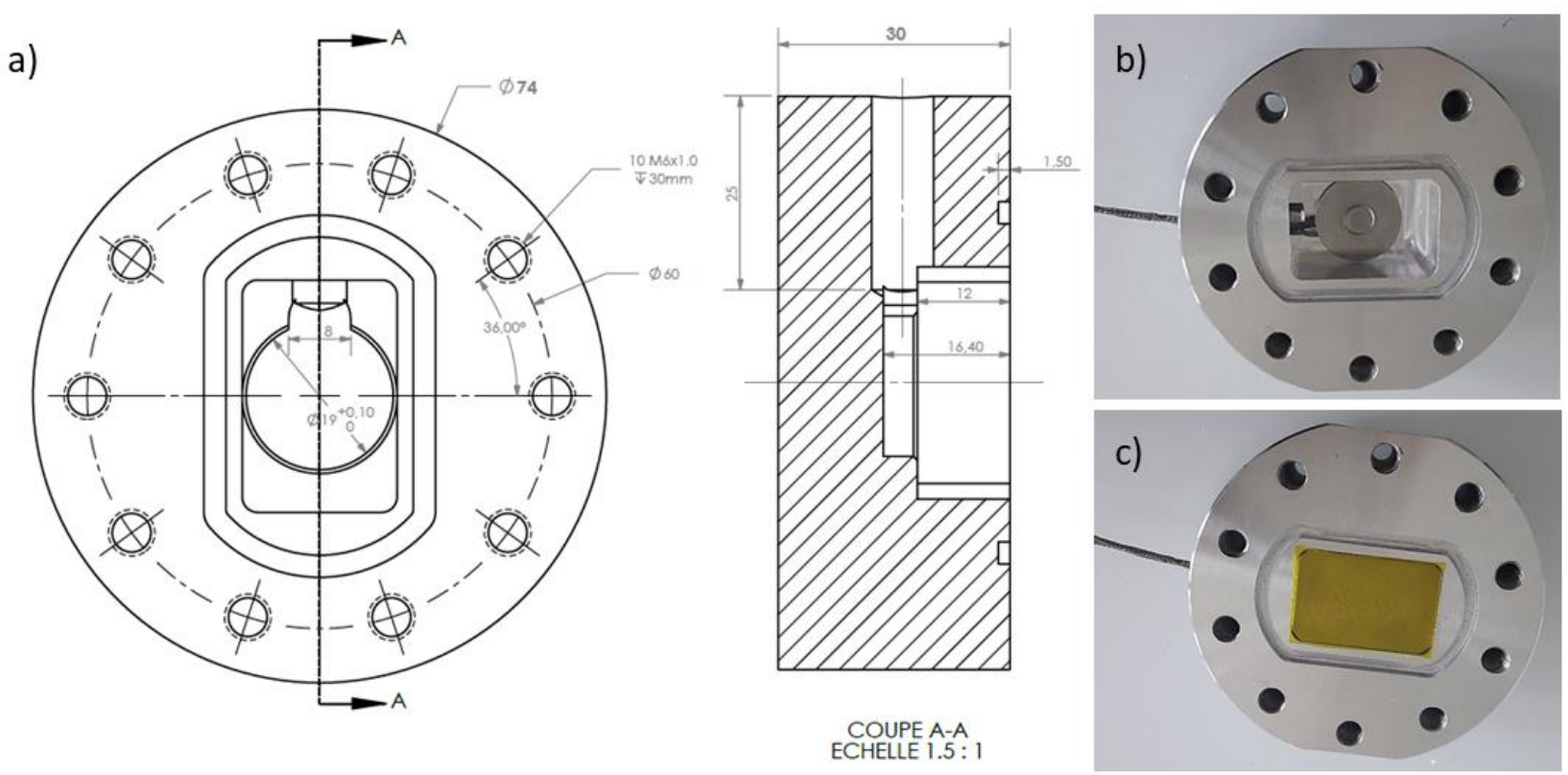

Figure 3: a) Drawings of the 7075 aluminium cell including the LCMKD-5KN load sensor b) Picture of the cell with the mounted sensor c) Picture of the cell including the aluminium flat plate and the PIM-1 sample

The measured compression loads (converted in compression pressure considering the PIM-1 sample area) are compared to the calculated values (considering both sample and seal areas). The values can be calculated according to the relationship (taken from DIN 946/VDI 2230) between the tightening torque and the tension generated. The relationship is given below:

$$
T=F\left(\frac{p}{2 \pi}+0.583 d_{2} \mu_{t}+\frac{D}{2} \mu_{h}\right)
$$

with $T$ the input torque, $\mu_{t}$ the thread friction coefficient, $\mu_{h}$ the underhead friction coefficient, $p$ the thread pitch, $D$ the effective diameter of the head contact, $d_{2}$, the effective diameter of thread contact and $F$ the tension generated during tightening. We assumed the friction under the head and the friction on the bearing surface of the screws being the same and equal to 0.15 (basic lubrication, screws used as received conditions, slightly oily). The others parameters were fixed according to M6 screws $\left(p=1 \mathrm{~mm} ; d_{2}=5.35 \mathrm{~mm}\right.$ and $D=6 \mathrm{~mm}$ ). The results obtained with the PIM-1 sample are presented in Table II and Figure 4.

We observe on Figure 4, above $4 \mathrm{Nm}$, the flattening of the pressure/load curve with the applied torque due to the increase of the sample and/or screws deformation. Indeed, plastic deformation of the screws seems to occur during the tightening process (screws A4-80 having a tensile strength of $800 \mathrm{MPa}$ ). We checked that the film deformations were elastic and reversible over the full torque range, since the measurements were reproducible. 
Table II: Calculated and measured compression pressures. The grey zone corresponds to the plateau values (see Figure 4 ) where the $P_{\text {calc }}$ is overestimated

\begin{tabular}{|c|c|c|c|c|c|c|c|c|c|c|}
\hline Load sensor used & \multicolumn{3}{|c|}{ LCMKD-5KN } & \multicolumn{7}{c|}{ LCM307-50KN } \\
\hline Torque [Nm] & $\mathbf{0 . 5}$ & $\mathbf{1 . 0}$ & $\mathbf{1 . 5}$ & $\mathbf{2}$ & $\mathbf{4}$ & $\mathbf{5}$ & $\mathbf{6}$ & $\mathbf{7}$ & $\mathbf{8}$ & $\mathbf{9}$ \\
\hline $\begin{array}{c}\text { Pcalc - calculated } \\
\text { pressure [bars] on the } \\
\text { sample and nitrile seal }\end{array}$ & 51 & 103 & 154 & 206 & 412 & 515 & 618 & 721 & 824 & 927 \\
\hline $\begin{array}{c}\text { Pmeas - measured } \\
\text { pressure [bars] on the } \\
\text { PIM-1 sample only }\end{array}$ & 42 & 87 & 127 & 198 & 402 & 455 & 470 & 482 & 486 & 488 \\
\hline
\end{tabular}

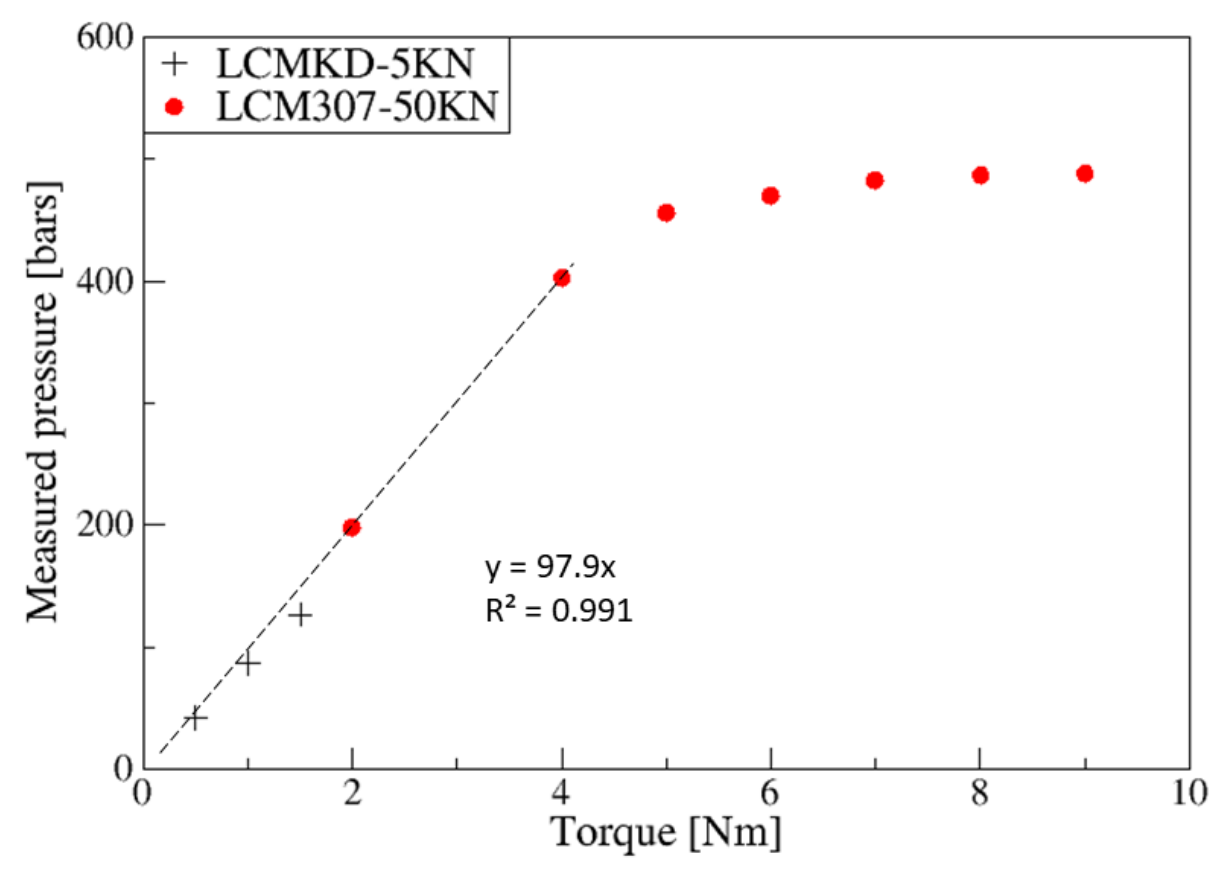

Figure 4: Experimentally extracted relationship between the torque and the load applied on the PIM-1 sample.

\subsection{Finite-element analysis of the stresses experienced by the sample}

To investigate the effect of the multi-axial loading due to the application of a uniaxial compression simultaneously to a hydraulic pressure gradient on the sample, a poromechanical simulation was undertaken with the finite-volume/finite-element open-source numerical solver BIL ${ }^{16}$. The sample properties are chosen according to the QENS measurements performed (PIM-1 sample, part 3): the PIM-1 film is $30 \mathrm{~mm}$ long and $300 \mu \mathrm{m}$ thick with a Young modulus of $10^{9} \mathrm{~Pa}$, a Poisson's coefficient of 0.4 and a Biot coefficient of 0.5 . The simulation consists in reproducing the steps of the experiment presented in section 3: the application, first, of a uniaxial compression of $80 \mathrm{MPa}$ with an initial hydraulic pressure of $0.1 \mathrm{MPa}$ (atmospheric pressure) in all the sample, and then the application 
of a hydraulic pressure gradient (10 MPa in the upstream surface and $0.1 \mathrm{MPa}$, atmospheric pressure, in the downstream surface). Results of the simulation are presented in Figure 5. Initially, the uniaxial compression ( $\sigma_{y y}$ on Figure $5 \mathrm{~b}$ ) is equal to $80 \mathrm{MPa}$ everywhere in the sample except at the extremities, which is an edge effect. We note that this compression, $\sigma_{y y}$, causes a stress in the perpendicular direction, $\sigma_{x x}$ on Figure 5a, resulting from the Poisson effect and the constrained displacement in the $\mathrm{x}$-direction. Upon applying the hydraulic pressure gradient and after reaching a steady state, the stress state is altered. It is observed that close to the zone where the water is injected, the $\sigma_{y y}$ stress rises progressively from -80 MPa to -84.9 MPa. Additionally, we observe a rise of $\sigma_{x x}$ in the film from 53.3 $\mathrm{MPa}$ to $-57.8 \mathrm{MPa}$ at the same sample positions. According to the results obtained, we consider that the alteration of the initial stress states $\left(\sigma_{x x}\right.$ and $\left.\sigma_{y y}\right)$ by the application of the hydraulic pressure gradient can be neglected when the applied hydraulic pressure is much smaller than the applied $\sigma_{y y}$ stress.
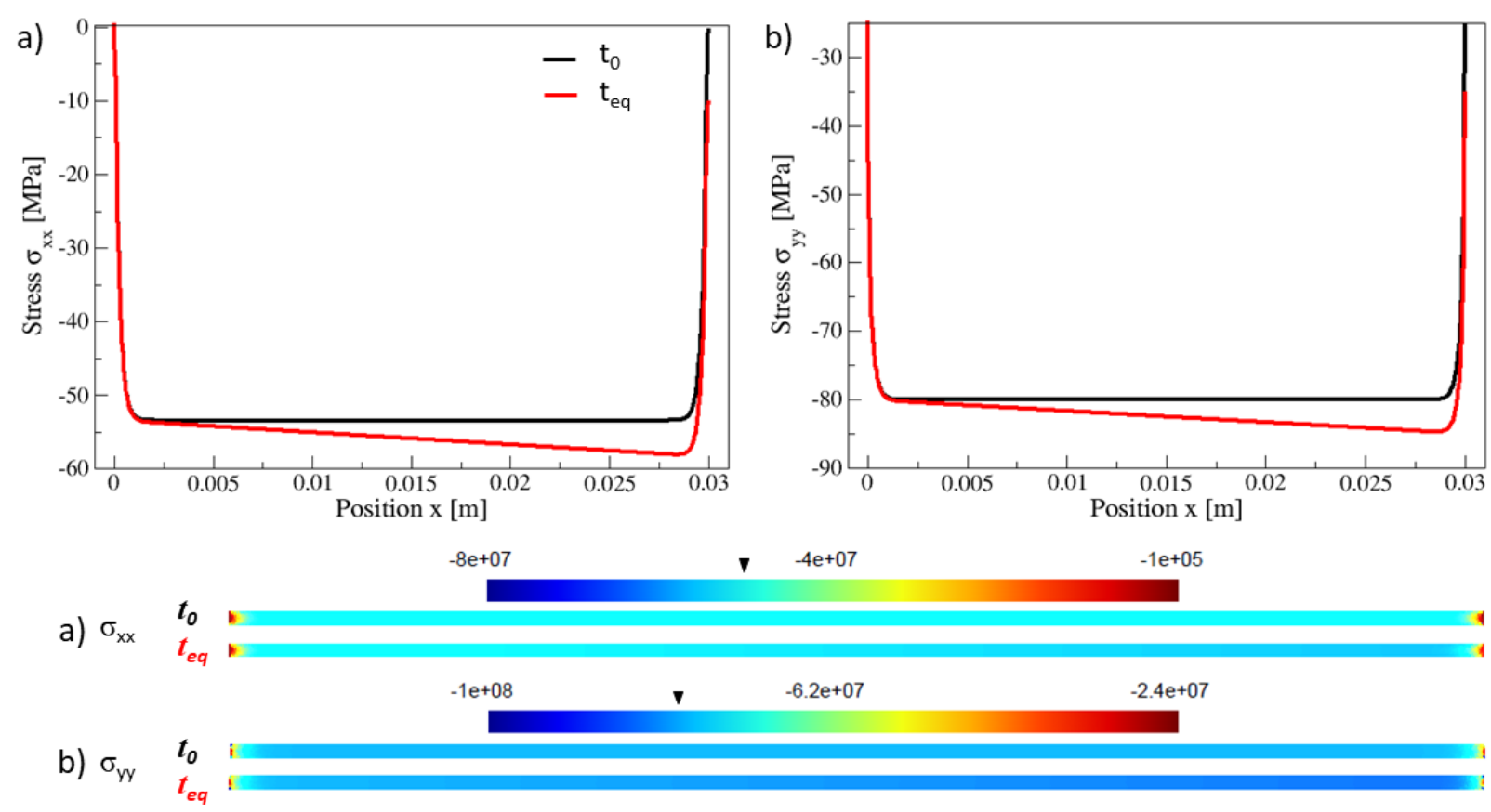

Figure 5: Stress state in the soft PIM-1 film before $\left(t_{0}\right)$ and after $\left(t_{\text {eq }}\right)$ the application of the hydraulic pressure gradient: a) $\sigma_{x x}$ distribution, stress on the axis of the film; $b$ ) $\sigma_{y y}$ distribution, stress perpendicular to the film. The stress distribution bars below show the stress state in a crosssection of the $30 \mathrm{~mm}$ and 300- $\mu \mathrm{m}$-thick film. The black cursors on the stress distribution bar caption indicate the initial stress state at the center of the film

\subsection{Cell characterization}

In the following section, we provide the characteristics of the sample holder through a neutron beam and compare it with other relevant holders. Measurements were performed with the following cells with no additional environment such as a cryostat.

- Our high-pressure (HP) cell: aluminium pressure-compression cell (Figure 1), $24 \mathrm{~mm}$ thick

- Cylindrical HP cell: cylindrical liquid pressure cell (ILL, 19PL10AL6, https://www.ill.eu/users/support-labs-infrastructure/sample-environment/equipment/highpressures/liquid-pressure-cells/) made of aluminium alloy 7049A-T6 with insert for flat 
sample, no mechanical compression here, only hydrostatic pressure (maximum pressure of 1 kbar)

- Flat slab: Standard aluminium flat slab

- TiZr HP cell: cylindrical liquid pressure cell (ILL, 13PL25TZ12, https://www.ill.eu/users/support-labs-infrastructure/sample-environment/equipment/highpressures/liquid-pressure-cells/) made from TiZr alloy with also an insert for flat sample (maximum pressure of $2.5 \mathrm{kbar}$ )

We observe on Figure $6 \mathrm{a}$ an increase of the elastic scattering intensity related to the high thickness of our HP cell. One should note that beside the height of the elastic contribution, an additional background is present in our cell (Figure 6b). Moreover, we can highlight that the cylindrical aluminium cell has small elastic and background contributions because of the favourable cell geometry for IN6 experiment (due to an almost constant scattering section with the scattering angle). However, beside its favourable geometry, the TiZr cell has a non-negligible elastic contribution because of the higher neutron absorption cross section of the material.
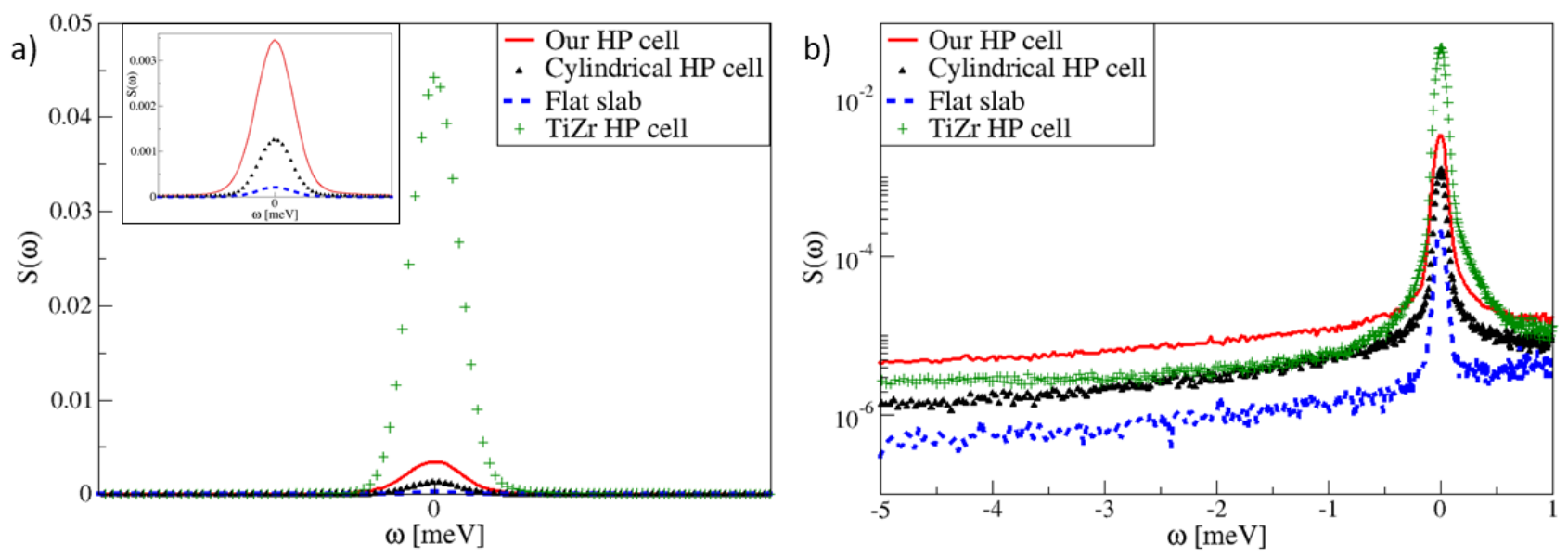

Figure 6: a) Plots of the $Q$-integrated spectra, $S(\omega)$, against energy transfer b) Log-plots to highlight the background contributions

As another important characteristic of the cell, we report on Figure 7 the extracted half width at half maximum (HWHM) from fitting data with a Lorentzian function. As expected, the broader HWHMs were observed for our HP cell and the flat slab, that have similar flat slab geometries, with an increase of the elastic width the scattering vector $(Q)$ because of the width of the holders. The HWHM variations of the two others cylindrical cells are constant with $Q$ thanks to the favourable geometry.

Finally, the Figure 8 shows the integrated intensity as a function of $Q$. The cylindrical cell obviously provides a much more favourable geometry enabling a smaller thickness. However, no uniaxial compression can be applied in such a cell. Moreover, using an insert with a slit for the sample necessarily creates a small water film around the insert. This bulk water acts as a background for the measurement of the confined water and may lead to impossible disentanglement of both signals. In our clamp-type flat cell, despite its very strong elastic signal, the absence of parasite QENS signal provides an invaluable benefit for the characterisation of the confined water. 


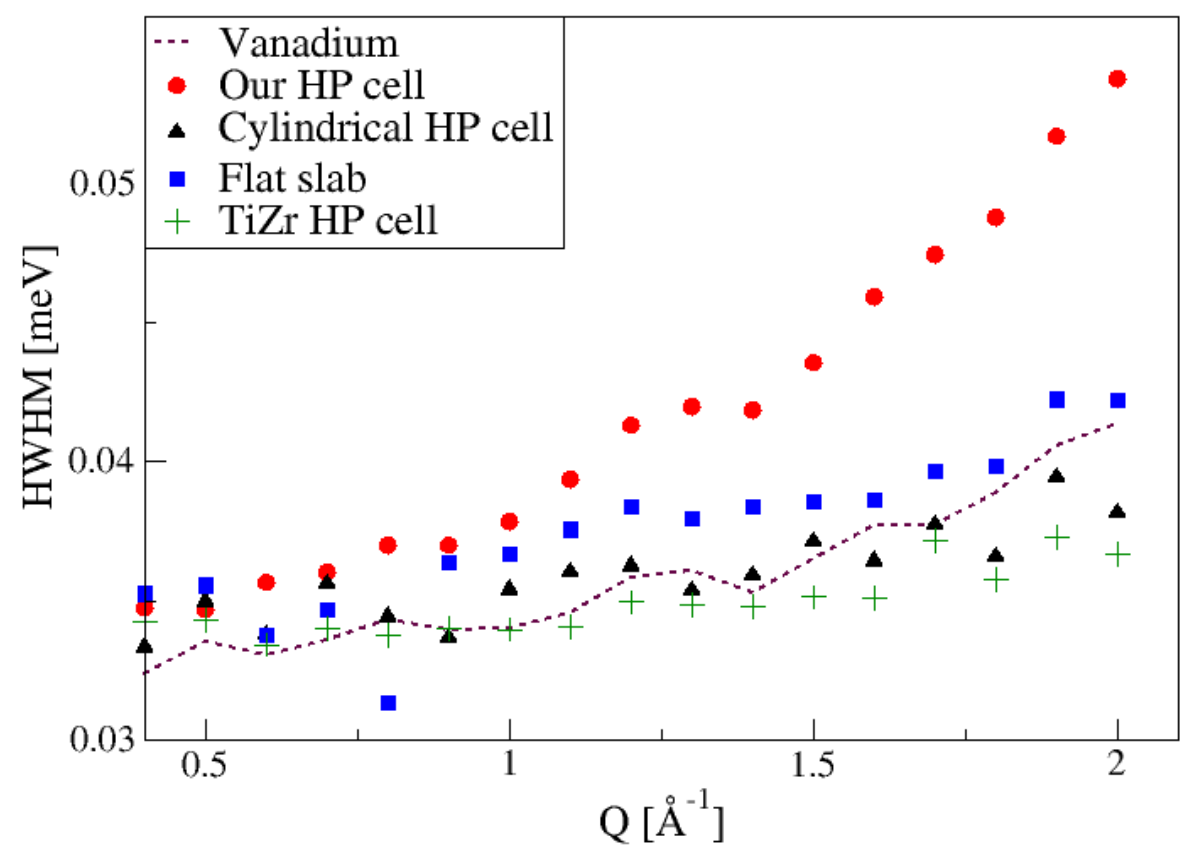

Figure 7: Variations of the extracted HWHM of the Lorentzian function using a flat background on the $-0.5 /+0.5$ meV energy range

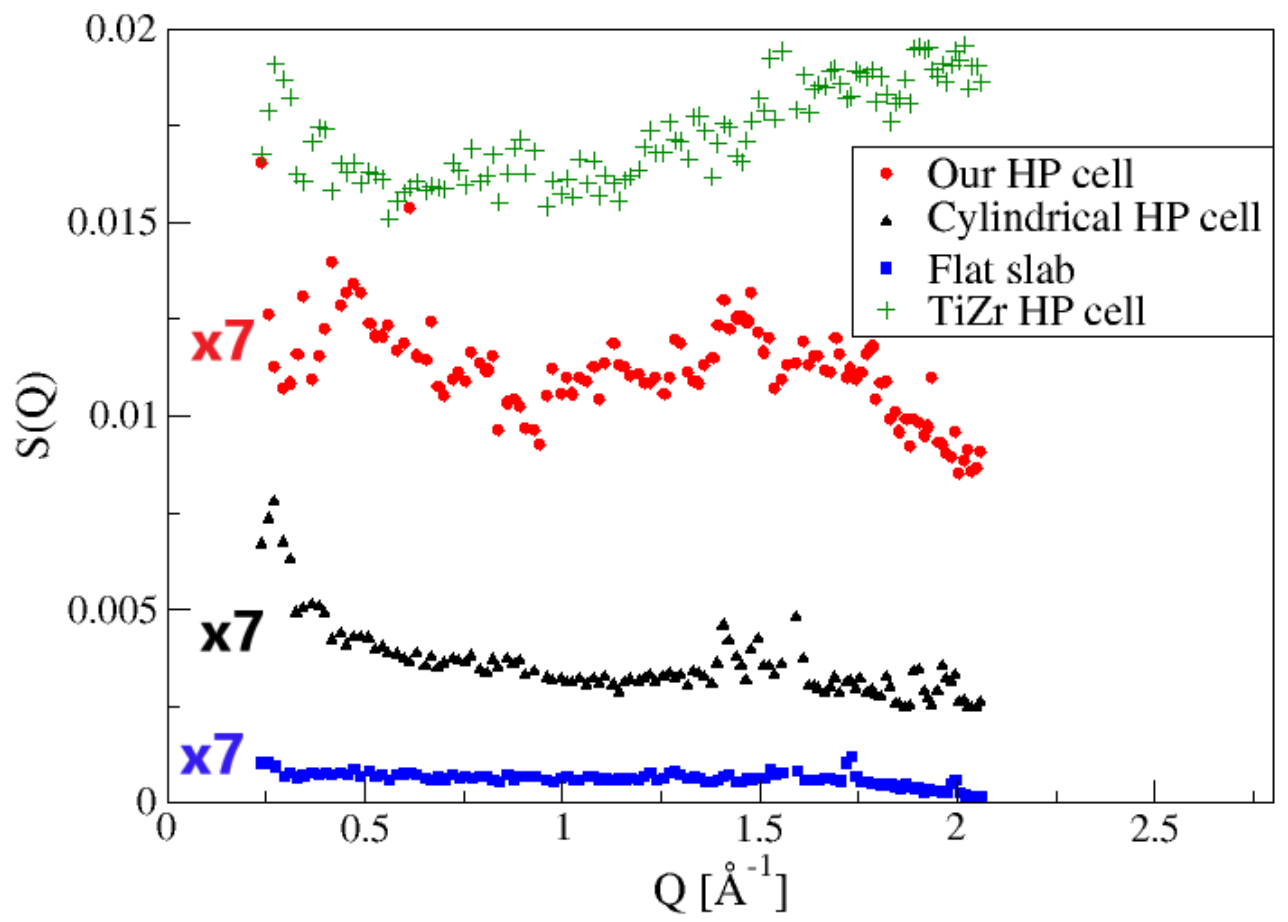

Figure 8: Plots of the $\omega$-integrated spectra, $S(Q)$, against the momentum transfer (sum across the channels around the elastic position). The scale of the $Y$-axis for the red, black and blue curves has been multiplied by 7 for clarity

\subsection{Description of the set-up}

Eventually, the description of the portable setup developed for measurements under hydraulic pressure gradient is described in Figure 9. The domestic pressure washer Karcher, giving high water flow (600 L/h maximum) and high pressure (up to 180 bars), is connected to the aluminum cell with stainless steel tubing (Figure 10). A by-pass valve is placed between the cell and the Karcher in order 
to control and stabilize the pressure of the inlet flow. This system enables moreover to bypass the internal security of the Karcher that stops the flow in case of restricted output diameter. Using the bypass valve, the water flows through the cell, creating a pressure gradient ( $P_{i n}=14-120$ bars) at the entrance of the cell and $P_{\text {out }}=P_{\text {atm }}$, at the output open on the water reservoir.

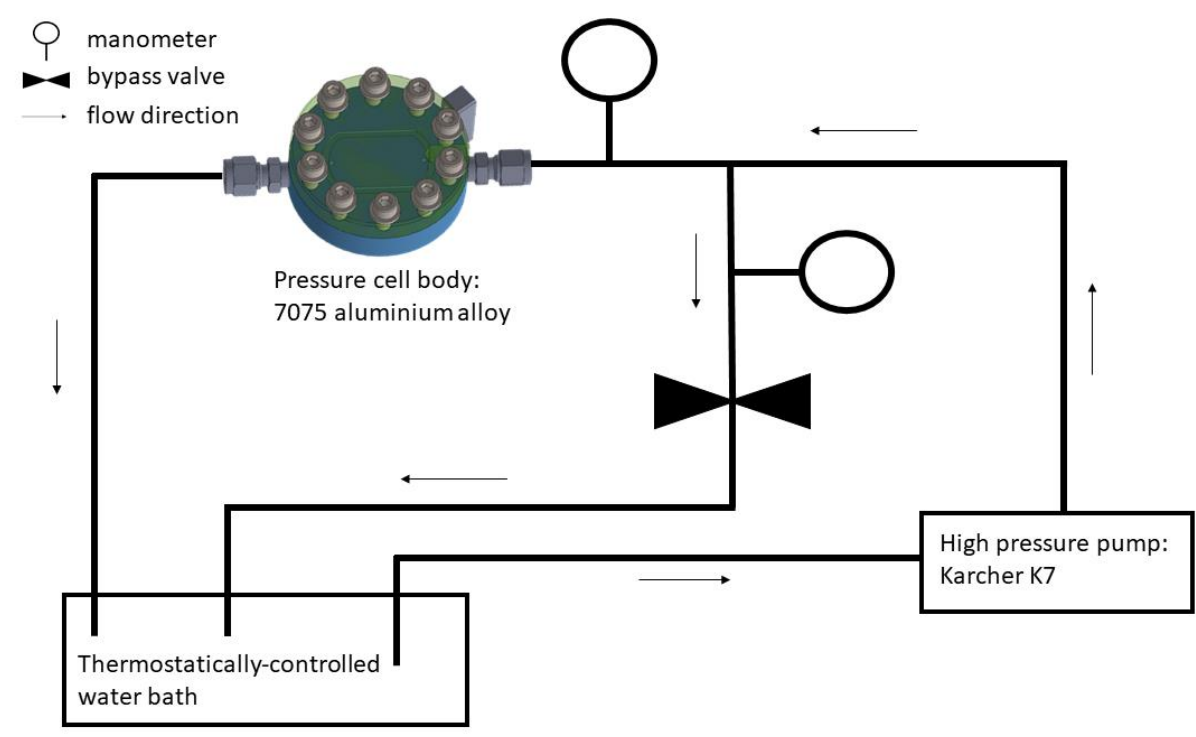

Figure 9: Setup
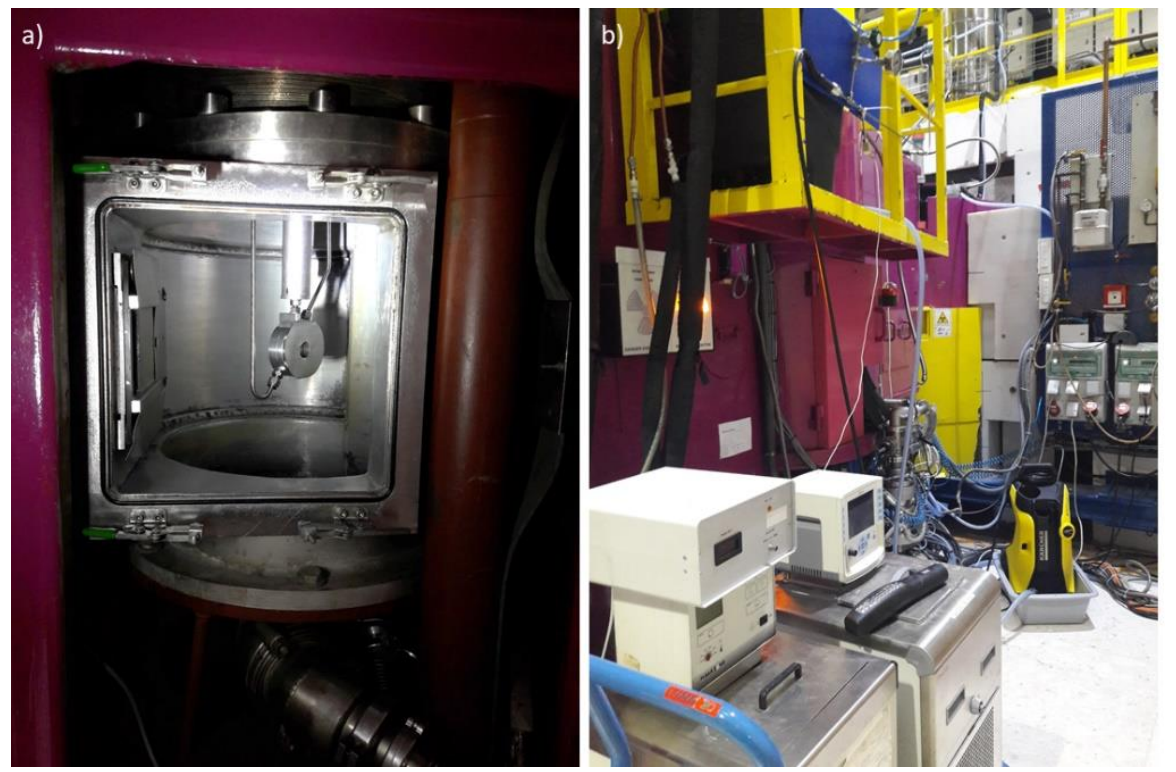

Figure 10: a) Picture of the HP cell mounted on the time-of-flight spectrometer, IN6, at the Institut Laue-Langevin (ILL) b) Picture of the Karcher connected to the HP cell. The flowing water is cooled by two thermostatically-controlled water baths

One considerable benefit from using a Karcher as a pressure pump is that the selected K7 model can be used in a closed-loop system proving a limited reservoir volume $(\sim 50 \mathrm{~L})$ that can be re-used infinitely, avoiding water wastage.

A drawback of the closed-loop system is that the circulating water can be considerably heated by the Karcher pump ( $3 \mathrm{~kW}$ ) and needs to be cooled with a powerful thermostatically-controlled water bath (Figure 10b). The water heating increases with the $\mathrm{P}_{\text {in }}$ pressure. The temperature of the water 
reservoir was monitored continuously and several improvements, like doubling the cooling power, increasing the water volume and stopping the Karcher (then the measurements) were used to maintain the temperature of the flowing water within $+/-3$ degrees of the measurement temperature.

\section{QENS measurements}

As a proof of concept, we present here QENS characterization of water confined in PIM-1 film. This polymer is made of rigid monomers that get entangled leading to nanometric pores within the bulk material. PIM-1 is made of a rather hydrophobic network (the water contact angle being $\left.95^{\circ}\right)^{17}$, so water penetrates only very slightly at atmospheric pressure.

\subsection{Materials and Methods}

The sample investigated is a Polymer of Intrinsic Microporosity (PIM-1) of $\sim 300 \mu \mathrm{m}$ thickness. PIM-1 was prepared and synthesized at the Institut Européen des Membranes (IEM). The material presents disordered interconnected pores of a few nanometers. Such microporous materials are widely investigated in the literature ${ }^{15,18-20}$ and can be used in a broad range of applications in industry (heterogeneous catalysis, adsorption, separation, and gas storage).

QENS experiments have been performed at $20^{\circ} \mathrm{C}$ on the time of flight neutron scattering spectrometer IN6-Sharp at the Institut Laue-Langevin (ILL) to access the water dynamics on the picosecond time scale. The incident neutron wavelength was $5.1 \AA$, corresponding to an elastic resolution of $\sim 100 \mu \mathrm{eV}$ and giving access to a $Q$-range of [0.4 - 2] $\AA^{-1}$.

The incoherent scattering function, $S(Q, \omega)$, is described by the convolution product in energy of the contributions of the translational $\left(S(Q, \omega)_{T}\right)$ and rotational $\left(S(Q, \omega)_{R}\right)$ motions of water. The vibrational motion is approximated through the Debye-Waller factor. The two others motions were coupled and described, respectively, with the jump diffusion model ${ }^{21}$ and the isotropic rotational diffusion in a sphere of radius $R^{22}$, roughly equal to the $\mathrm{O}-\mathrm{H}$ distance in a water molecule (1.0 $\AA$ ). We also included an elastic term represented by a delta function, $\delta(\omega)$, of area $A_{\text {elas }}(Q)$, and a flat background. The elastic term considers the aluminum cell and the dry PIM-1 contributions that were not subtracted from the signal. The general equation is given below:

$$
S(Q, \omega)=e^{\left(-\frac{\left\langle u^{2}\right\rangle Q^{2}}{3}\right)} \cdot\left(A_{\text {elas }}(Q) \cdot \delta(\omega)+A_{Q E N S}(Q) \cdot\left(S(Q, \omega)_{T} \otimes S(Q, \omega)_{R}\right)\right)
$$

with $\left\langle u^{2}\right\rangle$, the mean-square vibrational amplitude of hydrogen atoms.

The translational contribution can be written as:

$$
S(Q, \omega)_{T}=\frac{1}{\pi} \frac{\Gamma_{T}}{\omega^{2}+\Gamma_{T}^{2}}
$$

with $\Gamma_{T}$, the HWHM of the translational Lorentzian function. $\Gamma_{T}$ is defined by: 


$$
\Gamma_{T}=\frac{D_{T} Q^{2}}{1+\tau D_{T} Q^{2}}
$$

with $D_{T}$ and $\tau$, the self-diffusion constant and the residence time respectively. The rotational contribution is presented below:

$$
S(Q, \omega)_{R}=\mathrm{A}_{0}(Q) \cdot \delta(\omega)+\sum_{i=1}^{\infty} \mathrm{A}_{i}(Q) \cdot \frac{1}{\pi} \frac{\Gamma_{i}}{\omega^{2}+\Gamma_{i}^{2}}
$$

with

$$
\mathrm{A}_{i}(Q)=(2 i+1) j_{i}^{2}(Q R)
$$

and

$$
\Gamma_{i}=i(i+1) D_{R}
$$

$D_{R}$ is the isotropic rotational diffusion constant.

The experimental signal $S(Q, \omega)_{\text {meas }}$ is the convolution of the theoretical scattering law and the experimental resolution $R(Q, \omega)$ :

$$
S(Q, \omega)_{\text {meas }}=S(Q, \omega) \otimes R(Q, \omega)
$$

We note that the empty cell was chosen as the resolution for the fitting model, since its HWHM is increasing with $Q$ (see Figure 7) and measuring the vanadium sample (usually employed for the instrumental resolution) inside the cell was not appropriate for our system. Moreover, the multiple scattering contribution can be neglected since the coupled $\left\{\mathrm{PIM}-1\right.$ film/water $\left.\left(\mathrm{H}_{2} \mathrm{O}\right)\right\}$ transmission is close to 0.9 and the PIM-1 matrix only contributes to the elastic scattering, that is not analysed in this paper. Fitting of the data was performed on a restricted $Q$-range $\left(0.5\right.$ to $\left.1.6 \AA^{-1}\right)$ and energy range ($5 /+5 \mathrm{meV})$, and the Debye-Waller factor is calculated with $\left\langle u^{2}\right\rangle=0.5 \AA^{2}$.

\subsection{Results}

In this last section, the poromechanical coupling is investigated with the PIM-1 sample using QENS measurements. Figure 11 represents the sum over $Q, S(\omega)$, of the quasi-elastic spectra measured in different conditions of hydraulic pressure gradient and uniaxial compression. We can observe an important QENS signal in the PIM-1 spectra related to the mobility of the confined water in the nanopores. The dry PIM-1 indeed presents only elastic scattering. The two hydraulic pressure gradients, 14 and 85 bars, were applied using the set-up shown in Figure 9 together with three uniaxial compressions $(1 \mathrm{~N} / \mathrm{m}, 5 \mathrm{~N} / \mathrm{m}$ and $8 \mathrm{~N} / \mathrm{m})$ in the direction perpendicular to the water flow. According to the Table II, we note that $1 \mathrm{Nm}, 5 \mathrm{Nm}$ and $8 \mathrm{Nm}$ corresponds to $\sim 87$ bars, 455 bars and 486 bars respectively. 


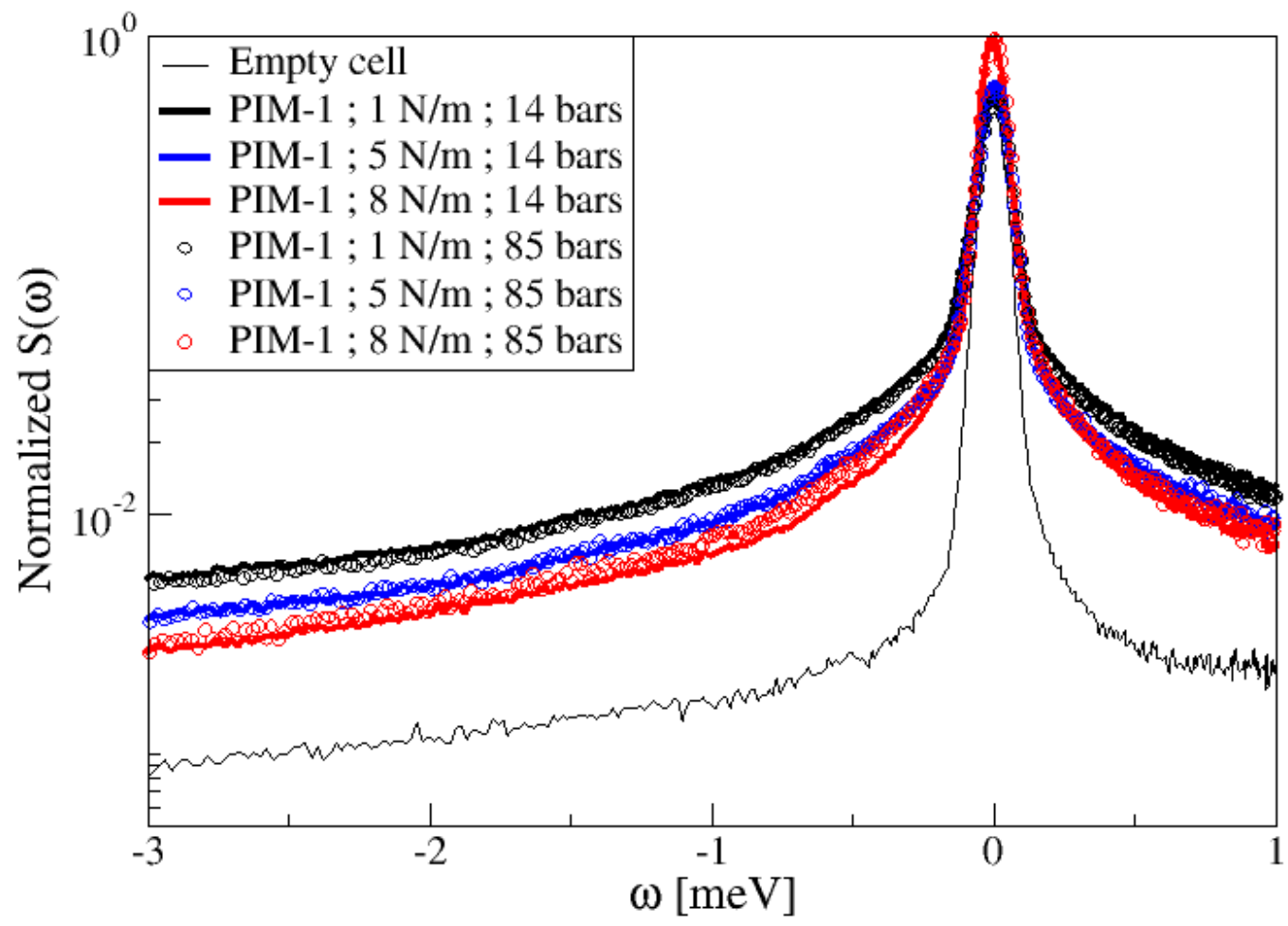

Figure 11: Quasi-elastic spectra of the empty cell and of the PIM-1 sample at three different uniaxial compressions and under constant hydraulic pressure gradients (14 bars and 85 bars) applied with the Karcher

The experimental data were fitted using the models presented in the method section. The obtained values of the different parameters are presented in Table III. Examples of fits at 14 bars, at two different $Q$-values and at two different uniaxial compressions are shown in Figure 12. Results show no influence of the hydraulic pressure gradient on the pressure scale investigated, but a clear dependence of the confined water dynamics with the uniaxial compression of the film.

a)

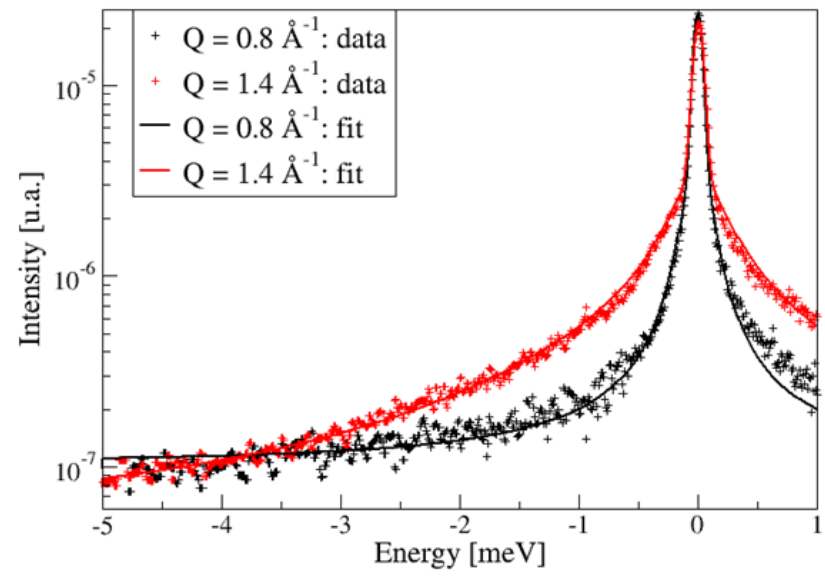

b)

14 bars $-8 \mathrm{Nm}$

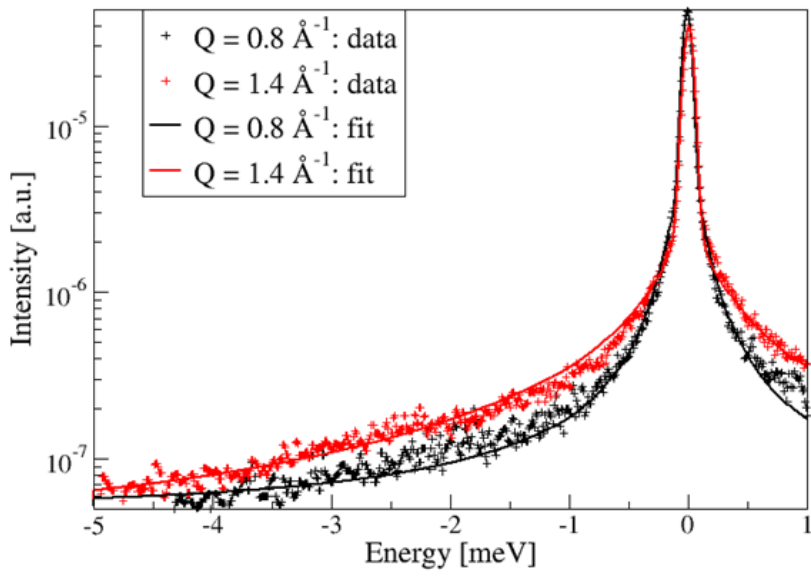

Figure 12: QENS spectra and the corresponding fits at $Q=0.9 \AA^{-1}$ and $1.4 \AA^{-1}$ under 14 bars of hydraulic pressure gradient at different uniaxial compressions a) $1 \mathrm{Nm}$ b) $8 \mathrm{Nm}$ 
Table III: Numerical values of the extracted fitting parameters.

\begin{tabular}{|c|c|c|c|c|}
\hline & & \multicolumn{2}{|c|}{ Translation: Jump diffusion model } & \multirow{2}{*}{$\begin{array}{c}\text { Rotational diffusion } \\
D_{R}[\mathrm{ps}] \text { - isotropic } \\
\text { rotational diffusion }\end{array}$} \\
\hline & & $\begin{array}{c}D_{T}\left[\mathrm{~cm}^{2} / \mathrm{s}\right]-\text { self } \\
\text { diffusion coefficient }\end{array}$ & $\begin{array}{c}\tau_{T}[\mathrm{ps}] \text { - } \\
\text { residence time }\end{array}$ & \\
\hline \multirow{3}{*}{$\begin{array}{c}14 \\
\text { bars }\end{array}$} & $1 \mathrm{Nm}$ & $(2.60 \pm 0.001) \mathrm{E}-05$ & $0.37 \pm 0.003$ & $0.73 \pm 0.001$ \\
\hline & $5 \mathrm{Nm}$ & $(2.90 \pm 0.01) \mathrm{E}-05$ & $0.81 \pm 0.01$ & $0.83 \pm 0.01$ \\
\hline & $8 \mathrm{Nm}$ & $(3.16 \pm 0.01) \mathrm{E}-05$ & $0.11 \pm 0.02$ & $0.91 \pm 0.02$ \\
\hline \multirow{3}{*}{$\begin{array}{c}85 \\
\text { bars }\end{array}$} & $1 \mathrm{Nm}$ & $(2.60 \pm 0.04) \mathrm{E}-05$ & $0.31 \pm 0.1$ & $0.70 \pm 0.04$ \\
\hline & $5 \mathrm{Nm}$ & $(2.87 \pm 0.1) \mathrm{E}-05$ & $0.37 \pm 0.1$ & $0.83 \pm 0.1$ \\
\hline & $8 \mathrm{Nm}$ & $(3.18 \pm 0.02) \mathrm{E}-05$ & $0.08 \pm 0.03$ & $0.85 \pm 0.02$ \\
\hline
\end{tabular}

The translational motion obtained at $1 \mathrm{Nm}$ is similar to that found for bulk water. Indeed, as presented in Table II, $1 \mathrm{Nm}$ corresponds to a compression pressure of $\sim 87$ bars which is of the same order of magnitude than the second tested hydraulic pressure gradient ( 85 bars). Thus, the water flow probably inhibits the compression effect, explaining why the translational motion is similar to the bulk dynamics. However, we observe an acceleration of the translational diffusion with the increase of the film compression. At $8 \mathrm{Nm}$ ( 486 bars $)$, the residence time significantly drops, indicating that the diffusion becomes closer to a Fickian law over molecular distances. The trends for the acceleration of the water dynamics is assigned to the hydrophobicity of the material. Indeed, the disruption of the water hydrogen bonded network close to the hydrophobic surface enables individual water molecules to move freely causing an acceleration of its motion ${ }^{23}$. The restriction of the confined space increases the water diffusivity. The rotational motion seems however not to be influenced.

\section{Conclusion}

In conclusion, we developed a new high-pressure cell for QENS experiments in order to study the poromechanical coupling in soft porous materials and also a suitable setup for measurements under pressure gradients up to 120 bars under water flow. This original setup comprising a Karcher for inducing the water flow is easily transportable and inexpensive. An experimental application on the PIM-1 soft nanoporous film were presented highlighting the feasibility and the reliability of the experiment. The background contributions due to the aluminum cell still allows the correct analysis of the confined water dynamics. Eventually, we highlighted a slight acceleration of the water confined in the hydrophobic matrix PIM-1 with the increase of its compression pressure. However, the 
hydraulic pressure gradient seems not to have any influence below 120 bars, which is the maximum pressure accessible with our setup.

\section{Acknowledgements}

The authors thank B. Coasne and J. Peters for fruitful discussions. Neutron beam time at the Institut Laue-Langevin is also gratefully acknowledged. The neutron data set is available at DOI: http://10.5291/ILL-DATA.CRG-2669. This work was supported by The French National Agency for Research (ANR - TWIST - 17-CE08-0003-01).

\section{Data Availability Statement}

The data that support the findings of this study are openly available in the ILL web portal at DOI: http://10.5291/ILL-DATA.CRG-2669.

\section{References}

${ }^{1}$ A. Taschin, P. Bartolini, A. Marcelli, R. Righini, and R. Torre, Faraday Discuss. 167, 293 (2013).

${ }^{2}$ S.-. C. J. Teixeira, J.-M. Zanotti M.-C. Bellissent-Funel, Phys. B 234-236, 370 (1997).

${ }^{3}$ I.M. Briman, D. Rébiscoul, O. Diat, J.M. Zanotti, P. Jollivet, P. Barboux, and S. Gin, J. Phys. Chem. C 116, 7021 (2012).

${ }^{4}$ B. Coasne, A. Galarneau, R.J.M. Pellenq, and F. Di Renzo, Chem. Soc. Rev. 42, 4141 (2013).

${ }^{5}$ C. Poinsignon and J.D.F. Ramsay, J. Chem. Soc. Faraday Trans. 1 Phys. Chem. Condens. Phases 82, 3447 (1986).

${ }^{6}$ M.C. Bellissent-Funel, S.H. Chen, and J.M. Zanotti, Phys. Rev. E 51, 4558 (1995).

${ }^{7}$ S. Mitra, R. Mukhopadhyay, I. Tsukushi, and S. Ikeda, J. Phys. Condens. Matter 13, 8455 (2001).

${ }^{8}$ P. Pissis and A. Kyritsis, J. Polym. Sci. Part B Polym. Phys. 51, 159 (2013).

${ }^{9}$ C.W. Macminn, E.R. Dufresne, and J.S. Wettlaufer, Phys. Rev. Appl. 5, 1 (2016).

${ }^{10}$ J.C. Perrin, S. Lyonnard, and F. Volino, J. Phys. Chem. C 111, 3393 (2007).

${ }^{11}$ S. Spagnoli, I. Morfin, M.A. Gonzalez, P. Çarçabal, and M. Plazanet, Langmuir 31, 2554 (2015).

${ }^{12}$ P. Kneisel, B. Lewis, and L. Turlington, Part. Accel. 53, 97 (1996).

${ }^{13}$ G. Lappegard and J. Kohler, Ann. Glaciol. 40, 37 (2005).

${ }^{14}$ D. Sertore, M. Fusetti, P. Michelato, C. Pagani, and P. Pierini, Proc. EPAC06, INFN MilanoLASA, Segrate 460 (2006). 
${ }^{15}$ N.B. Mc Keown and P.M. Budd, Chem. Soc. Rev. 35, 675 (2006).

${ }^{16}$ P. Dangla: Bil-2.6, a modeling platform based on finite/element methods (2018).

${ }^{17}$ Y. Lan, P. Peng, and P. Chen, Adv. Polym. Technol. 37, 3297 (2018).

${ }^{18}$ J. Weber, Q. Su, M. Antonietti, and A. Thomas, Macromol. Rapid Commun. 28, 1871 (2007).

${ }^{19}$ K. Polak-Kraśna, C. Fuhrhop, S. Rochat, A.D. Burrows, A. Georgiadis, C.R. Bowen, and T.J. Mays, Int. J. Hydrogen Energy 42, 23915 (2017).

${ }^{20}$ T. Emmler, K. Heinrich, D. Fritsch, P.M. Budd, N. Chaukura, D. Ehlers, K. Rätzke, and F. Faupel, Macromolecules 43, 6075 (2010).

${ }^{21}$ M. Bee, Quasielastic Neutron Scattering (Adam Hilger, United Kingdom, 1988).

${ }^{22}$ V.F. Sears, Can. J. Phys. 44, 1299 (1966).

${ }^{23}$ M.-J. Wei, J. Zhou, X. Lu, Y. Zhu, W. Liu, L. Lu, and L. Zhang, Fluid Phase Equilib. 302, 316 (2011). 